\title{
Regulatory perspective of biomarker bioanalysis during drug development
}

\author{
Chongwoo Yu*,1 \& E Dennis Bashaw ${ }^{1}$ \\ ${ }^{1}$ Office of Clinical Pharmacology, Office of Translational Sciences (OTS), Center for Drug Evaluation \& Research (CDER), US Food \& \\ Drug Administration, 10903 New Hampshire Avenue, Building 51, Room 2130, Silver Spring, MD 20993, USA \\ *Author for correspondence: Tel.: +1 301796 2335; chongwoo.yu@fda.hhs.gov
}

\section{"Reliable bioanalytical methods are essential as they are the firm foundation of drug development including the discovery and utilization of biomarkers."}

First draft submitted: 14 November 2018; Accepted for publication: 21 February 2019; Published online: 17 April 2019

Keywords: bioanalytical method validation (BMV) • biomarker • biomarker qualification

One of the most popular keywords used in drug development nowadays is 'biomarker'. Drug developers are searching for ways to expedite the drug discovery and development process while reducing the cost. The pharmaceutical industry has been shifting its drug development paradigm to focus on the 'patient-centered medicine' or 'personalized medicine' approach, aiming to deliver the right drug at the right dose to the right patient. Biomarkers can play a critical role in personalized medicine as an indicator of biological processes and have been used for decades, primarily to diagnose and prescribe treatment. For example: blood pressure reduction is a widely accepted surrogate end point for stroke prevention; and cholesterol levels have long been recognized as a surrogate end point for atherosclerotic disease. This raises the question of the difference between a 'surrogate end point' and a 'biomarker'. A biomarker is a defined characteristic that is measured as an indicator of normal biological processes, pathogenic processes or responses to an exposure or intervention, including therapeutic interventions [1]. Molecular (e.g., blood glucose), histologic, radiographic (e.g., tumor size), or physiologic (e.g., blood pressure) characteristics are types of biomarkers. Surrogate end points on the other hand are laboratory or physical signs that are used as a substitute for a clinically meaningful end point to predict how a patient feels, functions or survives and they are expected to predict the effect of the therapy [2]. Thus, surrogate end points are a subset of biomarkers. Biomarkers can be beneficial to many aspects of drug development. Biomarkers can be used in monitoring the safety of a therapy, determining if a treatment is having the desired effect on the body, predicting patients who might respond better to a drug from a safety or efficacy perspective. These utilities can potentially enable time and cost savings in clinical trials.

\section{Biomarker qualification}

Once a biomarker is identified, it needs to be clinically and analytically validated. Clinical validation ensures the biomarker reflects (identifies, measures, and predicts) the outcome of interest, while analytical validation ensures that the performance characteristics (e.g., accuracy, precision, selectivity, sensitivity, stability, and reproducibility) of the biomarker assay are fit-for-purpose. A lack of proper analytical validation can limit the utility of biomarker data and potentially drive an otherwise successful clinical development program into failure. Careful consideration of preanalytical factors, such as sample collection, shipment, tracking, storage and distribution, and analytical considerations including reference standard sourcing, sample preparation strategies, instrumentation platforms and the removal of matrix interferences are critical to ensure reliable biomarker quantitation in biological matrices.

From the US FDA's perspective, new biomarkers may be integrated into drug development through two review pathways. Traditionally, this occurred through the drug development process. During the drug development process, the drug developer can engage in discussion and reach an agreement with the FDA review staff to use a particular biomarker in a specific drug development program. Recently, the FDA has adopted a new mechanism called the Biomarker Qualification Program [3-5]. Qualification of a biomarker means that within a stated context of use, the 
biomarker has been demonstrated to reliably support a specified manner of interpretation and application in drug development. Once qualified, the biomarker information is made publicly available and may be used in multiple drug development programs under its qualified context of use [3-5].

\section{Biomarkers in the bioanalytical method validation guidance \& case examples}

For the rest of this article, focus will be on sharing the regulatory perspective on biomarker bioanalysis during drug development. For a biomarker to be used for regulatory decision-making, reliable measurement of biomarker concentrations is pivotal. A question that is often asked is 'how good is good enough?' The recently issued FDA's bioanalytical method validation (BMV) guidance outlines the Agency's recommendations on BMV to measure in vivo biomarker concentrations in biological matrices such as blood or urine. As biomarkers can be used for a variety of purposes during drug development, a fit-for-purpose approach should be used [6]. The answer to the question 'how good is good enough?' relies on the purpose of study being conducted and the question you are trying to answer. For instance, in the development program of sitagliptin, which is a novel dipeptidyl-peptidase IV (DPP4) inhibitor for Type 2 diabetes, plasma DPP4 activity was implemented as a target engagement biomarker [7]. The drug developer used DPP4 inhibition data for their proof of concept and dosage regimen selection for their Phase III clinical trials. An in-house bioanalytical method validation was used in their early development. While the demonstration of accuracy is required for BMV per the FDA's guidance, accuracy could not be determined due to the lack of defined quality controls (QCs) and pure standards for DPP4. However, the dosage regimen for the Phase III trial was selected based on the \% DPP4 inhibition data and this aided the drug developer in an internal decision-making process. While these data were important to the drug developer for internal decision making, it did not significantly contribute to the decision making about the overall efficacy and safety of the product. Therefore, the extent of BMV in this case was less critical for a regulatory decision. For biomarker assays used in early development (e.g., candidate selection, go/no-go decisions, or proof of concept), the drug developer should determine what level of BMV is appropriate and provides them with sufficient confidence for making internal decisions.

In a development program where Drug X suppresses Hormone A and Hormone A serves as a surrogate end point for the drug treatment, for Hormone A it was found that more than $33 \%$ of QCs were inaccurate in a large percentage of runs and more than $25 \%$ of calibrators were inaccurate for standard curves in the drug developer's BMV report. In addition, stability of Hormone A was not demonstrated adequately and as a result "degraded samples could result in being false positives, as lower concentrations of the Hormone A were desirable for demonstrating therapeutic benefit." In the end, it was concluded that the bioanalytical method could not accurately determine the true concentrations of Hormone A (i.e., biomarker) and this affected the efficacy determination. As the concentration of Hormone A determines the approvability of the drug (i.e., primary efficacy end point), the bioanalytical method measuring the concentration of Hormone A was critical for this application. Upon completion of its review, the FDA concluded that the data were unreliable and the application was not approved. As stated in the FDA's BMV guidance, "when biomarker data will be used to support regulatory decision-making, such as the pivotal determination of safety and/or effectiveness or to support dosing instructions in product labeling, the assay should be fully validated."

It should be noted that there are some occasions where commercial diagnostic kits (e.g., $510[\mathrm{k}]$ diagnostic assays) are utilized as analytical methods to measure concentrations of drugs, therapeutic biologics or biomarkers in development rather than the original intention for point-of-care patient diagnosis. For example, Drug Y was being developed as an oral drug and the concentration of Biomarker B was expected to be raised as a result of treatment with Drug Y. In this case, the concentration of Biomarker B was the primary efficacy end point. While an LC-MS/MS method was used for bioanalysis of Drug Y, an FDA-approved 510(k) competitive immunoassay was used for measurements of Biomarker B. However, it was found that the drug developer did not conduct full BMV for Biomarker B for use in drug development (rather than a point-of-care patient diagnosis) prior to use. There were no precision or accuracy assessments using human serum QC samples reported in the pivotal Phase III trial. It turned out that the actual QC control concentrations were unknown and reported in ranges rather than exact concentrations. There were no long-term, freeze-thaw, bench-top stability assessments reported for Biomarker B in human serum. In addition, the bioanalytical study (performance) report for the pivotal Phase III trial samples was not submitted to the new drug application. In their new drug application, the drug developer stated that their active ingredient is Drug Y, not Biomarker B and, therefore, they have not performed extensive testing on methods used to measure Biomarker B in human serum samples. The lack of supporting method validation and study 
performance data generated using the drug developer's bioanalytical method for its intended use raised a question on the reliability of measured Biomarker B concentrations for the pivotal Phase III clinical trial. Upon completion of its review, the FDA concluded that the effect of Drug Y on raising serum concentrations of Biomarker B in the pivotal Phase III clinical trial cannot be assessed as the drug developer's bioanalytical method was developed for point-of-care patient diagnosis rather than accurate and precise quantitation for use in drug development.

This latter situation, of repurposing an assay originally intended for a nonregulatory purpose, is a continuing problem in drug development and is potentially holding back future advances in the use of biomarkers. While at first it may seem 'easy' to take an 'off-the-shelf' point-of-care kit and repurpose it for drug development, as has been mentioned above, such an approach does not lessen the need for bioanalytical validation. Ultimately, the question comes down to how well one knows or understand the test and the inherent variability contained within an off-the-shelf test. To the regulator, submission of the standard curve or assay precision data from the kit labeling, which was based on work that may have been conducted numerous years ago in a vastly different setting, is insufficient in addressing the need to understand the performance of the test in the drug development setting. Going forward, the development of specific biomarker tests or assays and their validation for use (fit-for-purpose) needs to proceed hand-in-hand with the introduction of biomarkers in early clinical development. Point-of-care assays can be used in the exploratory stage but for refinement of the biomarker and its ultimate use to support regulatory decisions such as drug approvals and patient selection or monitoring, robust and reproducible performance must be demonstrated.

\section{Bioanalysis: the firm foundation of drug development}

To have confidence in the clinical implication of the data, we need to have confidence in the concentration values of target analyte(s), including biomarkers, and in order to have confidence in the concentration values, we must have confidence in the bioanalytical methods that are being used [8]. Reliable bioanalytical methods are essential as they are the firm foundation of drug development including the discovery and utilization of biomarkers. Failure to address this will prolong drug development, increase the cost, and cause the discarding of potentially valuable drugs due to a poor validation scheme. This is a situation that is totally avoidable with a holistic approach of having adequate data supporting bioanalytical method validation and biomarker performance (i.e., fit-for-purpose) that is both an indicator of and predictive of normal biological or pathogenic processes, or responses to an exposure or intervention, including therapeutic interventions [9].

\section{Disclaimer}

The views expressed are those of the authors and do not reflect official policy of the FDA. No official endorsement by the FDA is intended or should be inferred.

\section{Financial \& competing interests disclosure}

The authors have no relevant affiliations or financial involvement with any organization or entity with a financial interest in or financial conflict with the subject matter or materials discussed in the manuscript. This includes employment, consultancies, honoraria, stock ownership or options, expert testimony, grants or patents received or pending, or royalties.

No writing assistance was utilized in the production of this manuscript.

\section{References}

1. US FDA. CDER Biomarker Qualification Program (2018). www.fda.gov/Drugs/DevelopmentApprovalProcess/DrugDevelopmentTool sQualificationProgram/BiomarkerQualificationProgram/ucm535922.htm\#what-is

2. Temple RJ. A regulatory authority's opinion about surrogate end points. In: Clinical Measurement in Drug Evaluation. Nimmo WS, Tucker GT, (Eds). Wiley, NY, USA (1995).

3. US FDA. CDER Biomarker Qualification Program (2018). www.fda.gov/Drugs/DevelopmentApprovalProcess/DrugDevelopmentTool sQualificationProgram/BiomarkerQualificationProgram/ucm535882.htm

4. US FDA. Guidance for Industry and FDA Staff: Qualification for Drug Development Tools (2014). www.fda.gov/downloads/Drugs/GuidanceComplianceRegulatoryInformation/Guidances/UCM230597.pdf

5. Amur S, LaVange L, Zineh I, Buckman-Garner S, Woodcock J. Biomarker qualification: toward a multiple stakeholder framework for biomarker development, regulatory acceptance, and utilization. Clin. Pharmacol. Ther. 98(1), 34-46 (2015).

6. US FDA. Guidance for Industry: Bioanalytical Method Validation (2018). www.fda.gov/downloads/Drugs/GuidanceComplianceRegulatoryInformation/Guidances/UCM070107.pdf 
7. Krishna R, Herman G, Wagner JA. Accelerating drug development using biomarkers: a case study with sitagliptin, a novel DPP4 inhibitor for Type 2 diabetes. AAPS J. 10(2), 401-409 (2008).

8. Yu C, Bashaw ED. Regulatory perspective of bioanalysis from a clinical pharmacology reviewer standpoint: do you see what I see? Bioanalysis 6(4), 505-510 (2014).

9. BEST glossary (2018). www.ncbi.nlm.nih.gov/books/NBK338448/ 